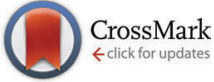

Cite this: Phys. Chem. Chem. Phys., 2017, 19, 204

Received 10th October 2016, Accepted 15th November 2016 DOI: $10.1039 / c 6 c p 06941 j$

www.rsc.org/pccp

\section{A butterfly motion of formic acid and cyclobutanone in the 1:1 hydrogen bonded molecular cluster}

\author{
Luca Evangelisti, ${ }^{a}$ Lorenzo Spada, ${ }^{a}$ Weixing Li, ${ }^{a}$ Susana Blanco, ${ }^{b}$ Juan Carlos López, ${ }^{b}$ \\ Alberto Lesarri, ${ }^{\text {b }}$ Jens-Uwe Grabow ${ }^{c}$ and Walther Caminati*a

\begin{abstract}
Upon supersonic expansion, formic acid and cyclobutanone (CBU) form a molecular cluster in which the two constituent molecules, linked by $\mathrm{OH} \ldots \mathrm{O}$ and $\mathrm{CH} \ldots \mathrm{O}$ hydrogen bonds, undergo a rapid interconversion between two equivalent forms. The tunneling motion takes place through the rupture and reformation of the $\mathrm{C}-\mathrm{H} \ldots \mathrm{O}$ hydrogen bond between the carbonyl oxygen of $\mathrm{HCOOH}$ and one of the two hydrogen atoms of the methylenic group adjacent to the cyclobutanone keto group. From the microwave spectra, tunneling energy splittings $\left(\Delta E_{01}\right)$ have been determined for the parent $(1122.756(3) \mathrm{MHz})$, DCOOH ....CBU (1084.538(1) MHz) and HCOOD ....CBU (1180.282(4) MHz) isotopic species. From these splittings, the potential barrier to interconversion has been calculated to be $B_{2}=39.7(5) \mathrm{cm}^{-1}$. The tunneling pathway is an asymmetric butterfly-like motion between the two moieties of the adduct, with a barrier at a configuration in which the ring plane of cyclobutanone is coplanar with formic acid.
\end{abstract}

\section{Introduction}

A number of complexes of carboxylic acids have been investigated by high resolution spectroscopy, providing information on the nonbonding interactions which link the constituent molecules, ${ }^{1-27}$ on the Ubbelohde effect related to the $\mathrm{OH} \rightarrow$ OD isotopic substitution, on occasionally occurring conformational equilibria, and - most noticeably - on the internal dynamics of large amplitude motions.

With respect to internal dynamics, useful information is obtained when splittings of the rotational transitions, due to tunneling motions connecting equivalent minima, are observed in high resolution (typically microwave) spectra. From these splittings it is generally possible to determine the potential energy barrier connecting equivalent minima. This kind of splitting has been measured for internal motions such as: (i) double proton transfer in dimers of carboxylic acids with a suitable symmetry; ${ }^{3-7}$ (ii) internal rotation of a light symmetric group (i.e. a $\mathrm{CH}_{3}$ group); ${ }^{1,5,18}$ (iii) inversion of an asymmetric

\footnotetext{
a Dipartimento di Chimica "G. Ciamician" dell'Università, Via Selmi 2, I-40126 Bologna, Italy. E-mail: walther.caminati@unibo.it

${ }^{b}$ Departamento de Química Física y Química Inorgánica, Facultad de Ciencias, Universidad de Valladolid, E-47011 Valladolid, Spain. E-mail: lesarri@qf.uva.es ${ }^{c}$ Institut für Physikalische Chemie und Elektrochemie, Lehrgebiet A,

Gottfried-Wilhelm-Leibniz-Universität, Callinstrasse 3A, D-30167 Hannover, Germany $\dagger$ Electronic supplementary information (ESI) available: Tables S1-S5 with the rotational transition frequencies and the MP2/6-311++G(d,p) optimized structure of the complex. See DOI: 10.1039/c6cp06941j
}

group (e.g. a hydroxyl or carboxylic acid) connecting two equivalent minima ${ }^{11,12,14,19}$ or (iv) internal motion of water in hydrated forms of carboxylic acids. ${ }^{15-21}$

Here we report the direct observation in the jet-cooled spectra of huge rotational splittings arising from a relative motion of a partner as heavy as cyclobutanone (CBU) with respect to formic acid (FA) in their 1:1 complex (FA-CBU). The tunneling pathway and the potential energy function of the motion have been derived from the experimental values for the $\Delta E_{01}$ splittings of some isotopologues, as described below.

\section{Methods}

\section{Experimental}

Samples of HCOOH, HCOOD, DCOOH and CBU were obtained commercially (Aldrich) and used without further purification. The rotational spectra of the FA . . CBU isotopologues have been recorded in two different laboratories:

(a) Bologna. Measurements were performed between 6 and 18.5 GHz using a high-Q pulse-excitation Fourier transform microwave (FTMW) spectrometer described elsewhere, ${ }^{28}$ based on the pioneering designs of Flygare ${ }^{29}$ and Grabow. ${ }^{30}$ Adducts were formed by flowing helium ( $\sim 5 \mathrm{~atm})$ through two stainless steel reservoirs containing, respectively, CBU at room temperature, and FA (or isotopologues) at $273 \mathrm{~K}$, and running the resulting gaseous mixture through a pulsed nozzle. Rotational frequencies were determined after the Fourier transform of 8k-data point 
time domain free induction decays, recorded with 40 ns sampling intervals. The pulsed molecular beam was introduced along the axis of a Fabry-Pérot resonator. Consequently, each observed transition appeared as a Doppler doublet, and the rest frequency was determined as the arithmetic mean of the frequencies of the two Doppler components. The accuracy of frequency measurements is estimated to be better than $3 \mathrm{kHz}$.

(b) Valladolid. The rotational spectrum of the parent species was initially recorded with a broadband direct-digital chirppulse FTMW spectrometer covering the frequency range of 2-8 GHz, which follows Pate's design. ${ }^{31}$ In this spectrometer a $5 \mu$ s chirp pulse created using an arbitrary waveform generator is amplified to $20 \mathrm{~W}$ and radiated perpendicular to the propagation of the jet expansion through a horn antenna. A molecular transient emission spanning $40 \mu \mathrm{s}$ is then detected through a second horn, recorded with a digital oscilloscope and Fourier-transformed to the frequency domain. Sample preparation was similar to Bologna, with optimal conditions requiring a backing pressure of $c a$. $4 \mathrm{~atm}$ and neon as the carrier gas. The accuracy of the frequency measurements is better than $15 \mathrm{kHz}$.

\section{Computational}

Information on the most stable geometries, and conformational and dissociation energies of the dimer was predicted computationally using both density functional theory (B3LYP assisted with empirical dispersion terms using the Grimme D3 scheme) and ab initio methods (MP2). All calculations used Pople's 6-311++G(d,p) basis set and were implemented in the Gaussian 09 program. ${ }^{32}$

\section{Results and discussion}

To get an initial guess on the shapes and relative energies of the plausible isomers of FA-CBU, we performed geometry optimizations comparing the performance of the MP2 and B3LYP-D3 methods. The two subunits are expected to assume a nearly co-planar arrangement in the complex. MP2 predictions suggest two energetically close minima, with the CBU ring slightly distorted (up or down) from planarity, while the B3LYP-D3 calculation predicts a single minimum, with the CBU ring nearly planar in the complex. Actually, the ring puckering of isolated CBU has a double minimum potential energy function with a small barrier close to $5 \mathrm{~cm}^{-1}$ at the planar ring. ${ }^{33}$ In this case the ground vibrational state lies above the barrier, corresponding to an effective planar ring. It is well-known that MP2 tends to overestimate ring-puckering barriers while B3LYP underestimates them. In particular, MP2 suggests ring-puckering distortions when it is not the case (even for benzene). In addition, in previous investigations of complexes of CBU with water ${ }^{34}$ and trifluoromethane, ${ }^{35}$ MP2 suggested two conformers differing in the distortion of the CBU ring, but only one was found, with CBU effectively being planar. For this reason, we followed the prediction of the B3LYP-D3 calculation. The rotational constants, the values of the dipole moment components and the absolute energy for the most stable conformer are reported in Table 1.
Table 1 B3LYP-D3/6-311++G(d,p) shape, energy, spectroscopic constants and dipole moment components of the most stable form of FA...CBU

\begin{tabular}{ll}
\hline$A / \mathrm{MHz}$ & 4120 \\
$B / \mathrm{MHz}$ & 993 \\
$C / \mathrm{MHz}$ & 843 \\
$\mu_{\mathrm{a}}, \mu_{\mathrm{b}}, \mu_{\mathrm{c}} / \mathrm{D}$ & $3.0,0.7,0.7$ \\
Absolute energy $/ E_{\mathrm{h}}$ & -421.150179
\end{tabular}

The rotational spectrum was first recorded for the parent species with a chirp-excitation microwave spectrometer in the frequency range of 2-8 GHz. Unfortunately, the spectrum was weak and considerably perturbed in that frequency region. We could reliably assign three transitions, $2_{02} \leftarrow 1_{01}, 3_{03} \leftarrow 2_{02}$, and $4_{04} \leftarrow 3_{03}$, split into two component lines associated with the two inversion sublevels of the vibrational ground state. We then recorded the $6-18.5 \mathrm{GHz}$ region using the pulse-excitation microwave spectrometer. We measured $31 \mu_{\mathrm{a}}$ - and $6 \mu_{\mathrm{b}}$-type transitions, displaying two intra-state tunneling components split up to $10 \mathrm{MHz}$. Finally, once good spectral predictions were iteratively obtained, 6 additional $\mu_{\mathrm{c}}$-transitions were measured, with the two components evenly spaced by $c a$. $2245 \mathrm{MHz}$. The latter lines were identified as inter-state transitions, so the separation between the two components is about the double of the energy separation of the two inversion sublevels.

All rotational transitions have been satisfactorily fitted using the following two-state coupled Hamiltonian:

$$
H=\left(\begin{array}{cc}
H_{0}^{\mathrm{R}}+H^{\mathrm{CD}} & H^{\text {int }} \\
H^{\text {int }} & H_{1}^{\mathrm{R}}+H^{\mathrm{CD}}+\Delta E_{01}
\end{array}\right)
$$

where $H_{0}^{\mathrm{R}}$ and $H_{1}^{\mathrm{R}}$ are the rotational terms for the 0 and 1 inversion sub-states, respectively, and $H^{\mathrm{CD}}$ represents the centrifugal distortion corrections (assuming the same value for the two inversion sub-states). The coupling term between the $v=0$ and $v=1$ states is expressed as:

$$
H^{\mathrm{int}}=F_{\mathrm{bc}} \times\left(P_{\mathrm{b}} P_{\mathrm{c}}+P_{\mathrm{c}} P_{\mathrm{b}}\right)+F_{\mathrm{ac}} \times\left(P_{\mathrm{a}} P_{\mathrm{c}}+P_{\mathrm{c}} P_{\mathrm{a}}\right)
$$

where $\Delta E_{01}$ is the energy difference between the two sub-states, and $F_{\mathrm{bc}}$ and $F_{\mathrm{ac}}$ are the Coriolis coupling parameters determined using the reduced axis system of Pickett, ${ }^{36}$ which are related to the off-diagonal elements of the inverse inertial tensor. The $\left(P_{\mathrm{a}} P_{\mathrm{b}}+P_{\mathrm{b}} P_{\mathrm{a}}\right)$ Coriolis term was not determinable from the fit, consistent with an inversion motion through an axis in the $a c$ inertial plane. Pickett's SPFIT program was used for the spectral analysis, ${ }^{37}$ using the $I^{\mathrm{r}}$-representation of Waltson's $S$ reduction. ${ }^{38}$ The resulting spectroscopic parameters are shown in the left column of Table 2 .

Additionally, we could assign the spectra of the two monodeuterated species DCOOH $\cdots$ CBU and HCOOD $\cdots$ CBU, using ca. 99\% enriched samples of DCOOH and HCOOD, respectively. The measured transition frequencies were fitted using the same procedure described for the parent species. The results of the fits are reported in the second and third columns of Table 2. No effects of the hyperfine quadrupole structure of the D atom 
Table 2 Experimental spectroscopic constants of the observed isotopologues of FA....CBU (Watson's S-reduction, $I^{r}$ representation)

\begin{tabular}{llll}
\hline & HCOOH $\cdots$ CBU & DCOOH $\cdots$ CBU & HCOOD $\cdots$ CBU \\
\hline$A_{0} / \mathrm{MHz}$ & $4151.912(9)^{a}$ & $4139.417(9)$ & $4119.26(1)$ \\
$A_{1} / \mathrm{MHz}$ & $4151.222(9)$ & $4138.707(10)$ & $4118.54(1)$ \\
$B_{0} / \mathrm{MHz}$ & $988.452(2)$ & $961.557(2)$ & $983.636(2)$ \\
$B_{1} / \mathrm{MHz}$ & $988.562(2)$ & $961.671(2)$ & $983.745(2)$ \\
$C_{0} / \mathrm{MHz}$ & $843.447(4)$ & $823.406(5)$ & $838.570(5)$ \\
$C_{1} / \mathrm{MHz}$ & $843.683(4)$ & $823.627(5)$ & $838.816(5)$ \\
$D_{\mathrm{J}} / \mathrm{kHz}$ & $0.596(1)$ & $0.565(2)$ & $0.586(1)$ \\
$D_{\mathrm{JK}} / \mathrm{kHz}$ & $-0.86(4)$ & $-1.04(7)$ & $-0.91(5)$ \\
$D_{\mathrm{K}} / \mathrm{kHz}$ & $11(1)$ & {$[11]^{b}$} & $11(2)$ \\
$d_{1} / \mathrm{kHz}$ & $0.039(1)$ & $0.037(2)$ & $0.040(1)$ \\
$d_{2} / \mathrm{kHz}$ & $-0.016(1)$ & $-0.016(5)$ & $-0.014(1)$ \\
$\Delta E_{01} / \mathrm{MHz}$ & $1122.756(3)$ & $1084.538(5)$ & $1180.282(5)$ \\
$F_{\mathrm{bc}} / \mathrm{MHz}$ & $5.2261(2)$ & $4.9179(7)$ & $5.2584(2)$ \\
$F_{\mathrm{ac}} / \mathrm{MHz}$ & $15.5(5)$ & $16.4(5)$ & $14.0(6)$ \\
$\sigma^{c} / \mathrm{kHz}$ & 5.1 & 5.2 & 4.5 \\
$N^{d}$ & 84 & 54 & 74
\end{tabular}

${ }^{a}$ Uncertainties in parentheses in units of the last digit. ${ }^{b}$ Values in brackets have been held fixed at the values of the parent species. ${ }^{c}$ Rootmean-square deviation of the fit. ${ }^{d}$ Number of transitions in the fit.

$(I=1)$ have been satisfactorily resolved. All the measured transition frequencies are given in the ESI $\dagger$ (Tables S1-S3).

Using the isotopic data, we report the Kraitchman substitution coordinates $^{39}$ of the two hydrogen atoms of the FA unit (H5 and H4) in Table 3. The atom numbering and the principal inertial axes system of the complex are shown in Fig. 1. The derived atomic positions are in good agreement with the theoretical values.

The B3LYP-D3/6-311++G(d,p) structures of the two equivalent energy minima and the transition state on the potential energy surface connecting the two minima (see Fig. 2 and the following paragraph) are reported in the ESI $\dagger$ (Tables S4 and S5). Adjusting the theoretical values of the structural parameters $r_{\mathrm{O} 6-\mathrm{O} 2}, \angle \mathrm{O} 6 \mathrm{O} 2 \mathrm{C} 1$ and $\angle \mathrm{O} 6 \mathrm{O} 2-\mathrm{C} 1 \mathrm{O} 3$ from $2.7475 \AA, 111.0^{\circ}$ and $-2.2^{\circ}$ to $2.7800 \AA$, $109^{\circ}$ and $0.4^{\circ}$, respectively, let us obtain an effective structure reproducing the experimental rotational constants within a few MHz. The bond lengths of the two hydrogen bonds corresponding to this geometry are: $r_{\mathrm{O} 6-\mathrm{H} 4}=1.790 \AA$ and $r_{\mathrm{O} 3-\mathrm{H} 16}=$ $2.464 \AA$, respectively. These values are also shown in Fig. 1 , and are in line with the values for "conventional" $\mathrm{O}-\mathrm{H} \cdots \mathrm{O}$ and weak $\mathrm{C}-\mathrm{H} \cdots \mathrm{O}$ hydrogen bonds.

The three determined splittings $\Delta E_{01}$ are related to the height $B_{2}$ of the potential barrier connecting the two equivalent minima

Table 3 Substitution $\left(r_{\mathrm{s}}\right)$, equilibrium $\left(r_{\mathrm{e}}\right.$, B3LYP-D3/6-311++G(d,p)) and effective $\left(r_{0}\right)$ atomic coordinates of the two substituted hydrogen atoms in the principal axes system of the parent species of FA ...CBU

\begin{tabular}{lllll}
\hline & & $r_{\mathrm{s}}$ & $r_{\mathrm{e}}$ & $r_{0}{ }^{a}$ \\
\hline $\mathrm{H} 4$ & $|a| / \AA$ & $1.584(2)^{b}$ & 1.4549 & 1.4991 \\
& $|b| / \AA$ & $0.989(3)$ & 1.0365 & 1.0635 \\
& $|c| / \AA$ & $0.06(5)$ & 0.1812 & 0.1806 \\
$\mathrm{H} 5$ & $|a| / \AA$ & $3.7785(8)$ & 3.7924 & 3.7720 \\
& $|b| / \AA$ & $0.579(6)$ & 0.5712 & 0.6365 \\
& $|c| / \AA$ & $0.22(2)$ & 0.0682 & 0.1247
\end{tabular}

${ }^{a}$ Calculated from the partial $r_{0}$ structure (see the text). ${ }^{b}$ Uncertainties in parenthesis in units of the last digit.

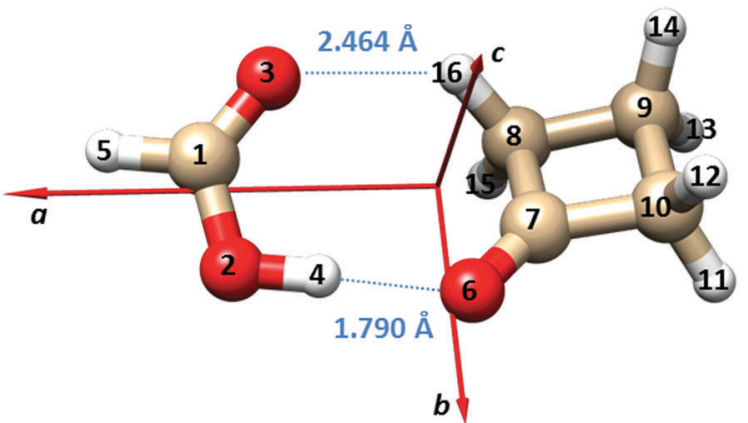

Fig. 1 Principal inertial axes and atom numbering of the observed dimer of FA ....CBU. The bond lengths of the two hydrogen bonds are also given.

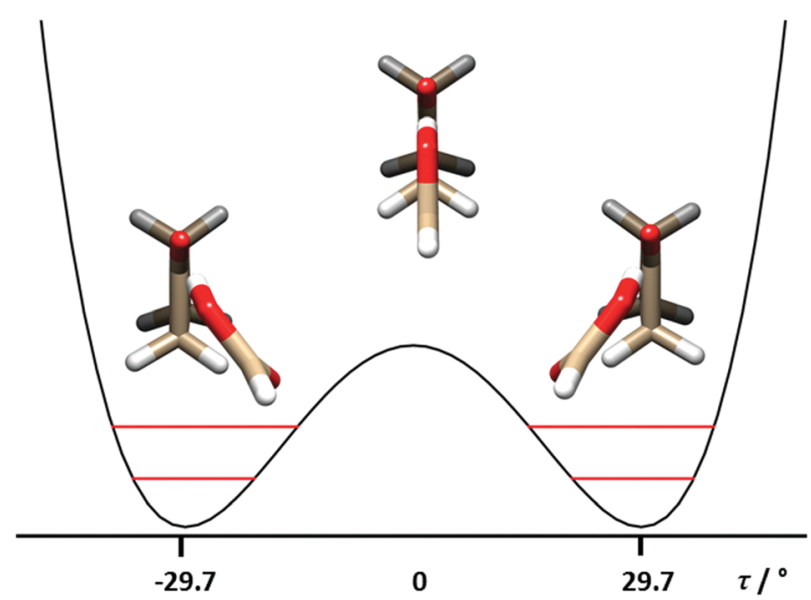

Fig. 2 The tunneling motion in $\mathrm{FA}-\mathrm{CBU}$ is due to the internal rotation around the C7O6-O2C1 $(\tau)$ dihedral angle, accompanied by structural relaxation of some other parameters.

depicted in Fig. 2, and to the specific pathway of the tunnelling motion. As expected, $\Delta E_{01}(\mathrm{DCOOH} \cdots \mathrm{CBU})=1084.538 \mathrm{MHz}$ is smaller than $\Delta E_{01}(\mathrm{HCOOH} \cdots \mathrm{CBU})=1122.756 \mathrm{MHz}$, because the reduced mass of the motion is higher for DCOOH $\cdots$ CBU. However, unexpectedly, $\Delta E_{01}(\mathrm{HCOOD} \cdots \mathrm{CBU})=1180.282 \mathrm{MHz}$ is larger than the value of the parent species. This is probably related to the Ubbelohde effect, ${ }^{40}$ often observed in the complexes of carboxylic acids, ${ }^{8-14}$ and related to the change in the bond length of the $\mathrm{O}-\mathrm{H} \cdots \mathrm{O}$ hydrogen bond upon $\mathrm{H} \rightarrow \mathrm{D}$ substitution.

For this reason, we relied only on the former two $\Delta E_{01}$ values to calculate the potential energy surface of the intermolecular motion. According to the theoretical calculations, $B_{2}$ is a function of the dihedral angle $\mathrm{C} 7 \mathrm{O}-\mathrm{O} 2 \mathrm{C} 1(\tau)$, which is the main parameter describing the relative motion of the two subunits. However, other structural parameters undergo considerable changes as a function of $\tau$, influencing the reduced mass of the motion. The latter depends not only on $\tau$ with respect to the rigid moieties, but also on the dynamic structural relaxation which takes place during the complex's large amplitude motion.

Meyer's flexible model ${ }^{41}$ is especially suitable to determine potential energy surfaces from rotational and vibrational experimental data. As justified by the theoretical predictions, we describe the large amplitude motion along the dihedral 
angle $\tau$ with any structural relaxations in other co-ordinates of the dimer constrained into a one-dimensional function of $\tau$. The double minimum potential can then be parameterized and described using the following function:

$$
V(\tau)=B_{2}\left[1-\left(\tau / \tau_{0}\right)^{2}\right]^{2}
$$

where the barrier $B_{2}$ at $\tau=0^{\circ}$ and the equilibrium value of the inversion angle $\tau_{0}$ (see Fig. 2) are the two parameters required. Being limited to two experimental splittings, we fixed $\tau_{0}$ at its B3LYP-D3/6-311++G(d,p) estimate $\left(29.7^{\circ}\right)$. Guided by the theoretical structural differences between the potential energy minimum $\left(\tau=\tau_{0}\right)$ and the top of the potential energy barrier $\left(\tau=0^{\circ}\right)$, we accounted for three structural parameters as a function of the dihedral angle $\tau$ as given in the following expressions (see Fig. 1 for labelling):

$$
\begin{aligned}
& \angle \mathrm{C} 8 \mathrm{C} 7-\mathrm{O} 6 \mathrm{O} 2 /^{\circ}=12.0 \cdot\left(\tau / \tau_{0}\right) \\
& \angle \mathrm{O} 6 \mathrm{O} 2-\mathrm{C} 1 \mathrm{O} 3 /^{\circ}=-2.2 \cdot\left(\tau / \tau_{0}\right) \\
& \angle \mathrm{C} 7 \mathrm{O} 6 \mathrm{O} 2 /^{\circ}=126.7-3 \cdot\left(\tau / \tau_{0}\right)^{2}
\end{aligned}
$$

However, when using these structural constraints in Meyer's one-dimensional flexible model, the ratio $\Delta E_{01}(\mathrm{HCOOH} \cdots \mathrm{CBU}) /$ $\Delta E_{01}(\mathrm{DCOOH} \cdots \mathrm{CBU})$ was very close to 1 rather than 1.035 . To be able to reproduce the right ratio, we had to modify the relaxation parameter of the $\mathrm{O} 6 \mathrm{O} 2-\mathrm{C} 1 \mathrm{O} 3$ dihedral angle from $-2.2^{\circ}$ to $+10^{\circ}$. In this way the ratio and values of the two experimental splittings were satisfactorily reproduced at a barrier $B_{2} \sim 39.7 \mathrm{~cm}^{-1}$. The results of the flexible model calculations are summarized in Table 4.

In the flexible model calculations, the $\tau$ coordinate has been probed in the $\pm 60^{\circ}$ range and solved into 41 mesh points. ${ }^{41}$ Going back to the HCOOD ...CBU isotopologue, a barrier of about $1 \mathrm{~cm}^{-1}$ lower is suitable to reproduce the $\Delta E_{01}$ splitting adhering to the parametrization used for the two former isotopologues. Using this model, the experimental ratios $F_{\mathrm{ac}} / F_{\mathrm{bc}}$ are also reproduced using the Eckart axis system of Pickett and Strauss. ${ }^{42}$

The positive value $\left(+10^{\circ}\right)$ of the relaxation parameter for O6O2-C1O3 suggests that the tunneling is due to an asymmetric butterfly motion of the two moieties with respect to each other, with the displacement with respect to the planarity of the O6 atom being much smaller that that of the C8 atom.

Table 4 Results of the flexible model calculations

\begin{tabular}{lll}
\hline Tunneling splittings & Obs. & Calc. \\
\hline$\Delta E_{01}(\mathrm{HCOOH} \cdots \mathrm{CBU}) / \mathrm{MHz}$ & 1122.8 & 1119.8 \\
$\Delta E_{01}(\mathrm{DCOOH} \cdots \mathrm{CBU}) / \mathrm{MHz}$ & 1084.5 & 1079.6 \\
\hline
\end{tabular}

Potential energy parameters

$\begin{array}{ll}B_{2}=39.7(5) \mathrm{cm}^{-1} & \tau_{0}=29.7^{\circ}\end{array}$

Structural relaxation parameters

$\angle \mathrm{C} 8 \mathrm{C} 7-\mathrm{O} 6 \mathrm{O} 2 /^{\circ}=12.0 \cdot\left(\tau / \tau_{0}\right)$

$\angle \mathrm{O} 6 \mathrm{O} 2-\mathrm{C} 1 \mathrm{O} 3{ }^{\circ}=10 \cdot\left(\tau / \tau_{0}\right)$

$\angle \mathrm{C} 7 \mathrm{O} 6 \mathrm{O} 2{ }^{\circ}=126.7-3.0 \cdot\left(\tau / \tau_{0}\right)^{2}$
The dissociation energy of the complex, $E_{\mathrm{D}}$, has been calculated at the MP2 and B3LYP-D3 levels to be $40.7 \mathrm{~kJ} \mathrm{~mol}^{-1}$ and $47.9 \mathrm{~kJ} \mathrm{~mol}^{-1}$, respectively. In order to obtain a comparable experimental estimate, it is necessary to approximate $E_{\mathrm{D}}$ from the centrifugal distortion parameter $D_{\mathrm{J}}$ within the so-called pseudo diatomic approximation ${ }^{43}$ combined with a LennardJones type potential. ${ }^{44}$ This approximation is valid when the intermolecular stretching motion leading to the dimer dissociation is almost parallel to the $a$-axis of the complex. In the present case such a procedure leads to an $E_{\mathrm{D}}$ value of $9.7 \mathrm{~kJ} \mathrm{~mol}^{-1}$, which is plausibly an underestimated value. While the theoretical values are not necessarily correct, the large discrepancy with the pseudodiatomic model results indicates that this model probably fails in this case, both because of the non-co-linearity of the dissociative stretching mode with the $a$-axis and the fact that $D_{\mathrm{J}}$ contains contributions from other vibrational motions.

\section{Conclusion}

The experimental observation of large tunneling splittings for three isotopologues of the adduct FA ...CBU allowed describing the inversion pathway and the potential energy surface along the C7O6-O2C1 dihedral angle. This is one of the first times that the relative motion of two relatively large molecules constituting a heterodimer is characterized quantitatively from the tunnelling splitting measured in the complex's rotational spectrum. The two subunits are linked through a conventional $\mathrm{O}-\mathrm{H} \cdots \mathrm{O}$ hydrogen bond, which acts as a fulcrum for a butterfly-like motion that lets the $\mathrm{O} 3$ oxygen alternate between a $\mathrm{C}-\mathrm{H} \cdots \mathrm{O}$ contact (or weak hydrogen bond) with the hydrogen atoms H15 or H16, corresponding to the two equivalent minima.

\section{Acknowledgements}

We thank the Italian MIUR (PRIN project 2010ERFKXL_001) and the University of Bologna (RFO) for financial support. We also thank funding from the Spanish MINECO-FEDER (CTQ201568148-C2-2-P) and the Junta de Castilla y León (UNVA-13-3E-2103).

\section{References}

1 L. Martinache, W. Kresa, M. Wegener, U. Vonmont and A. Bauder, Microwave Spectra and Partial Substitution Structure of Carboxylic Acid Bimolecules, Chem. Phys., 1990, 148, 129-140.

2 S. Antolinez, H. Dreizler, V. Storm, D. H. Sutter and J. L. Alonso, The Microwave Spectrum of the Bimolecule Trifluoroacetic Acid...Cyclopropanecarboxylic Acid, $Z$. Naturforsch., A: Phys. Sci., 1997, 52, 803-806.

3 I. Kalkman, C. Vu, M. Schmitt and W. L. Meerts, Tunneling Splittings in the $S_{0}$ and $S_{1}$ States of the Benzoic Acid Dimer Determined by High-Resolution UV Spectroscopy, ChemPhysChem, 2008, 9, 1788-1797.

4 A. M. Daly, K. O. Douglass, L. C. Sarkozy, J. L. Neill, M. T. Muckle, D. P. Zaleski, B. H. Pate and S. G. Kukolich, Microwave Measurements of Proton Tunneling and Structural 
Parameters for the Propiolic Acid-Formic Acid Dimer, J. Chem. Phys., 2011, 135, 154304.

5 M. C. D. Tayler, B. Ouyang and B. J. Howard, Unraveling the Spectroscopy of Coupled Intramolecular Tunneling Modes: A Study of Double Proton Transfer in the Formic-Acetic Acid Complex, J. Chem. Phys., 2011, 134, 054316.

6 G. Feng, L. B. Favero, A. Maris, A. Vigorito, W. Caminati and R. Meyer, Proton Transfer in Homodimer of Carboxylic Acids: The Rotational Spectrum of the Dimer of Acrylic Acid, J. Am. Chem. Soc., 2012, 134, 19281-19286.

7 L. Evangelisti, P. Ecija, E. J. Cocinero, F. Castaño, A. Lesarri, W. Caminati and R. Meyer, R. Proton Tunneling in Heterodimers of Carboxylic Acids: A Rotational Study of the Benzoic AcidFormic Acid Bi-Molecule, J. Phys. Chem. Lett., 2012, 3, 3770-3775.

8 G. Feng, Q. Gou, L. Evangelisti, Z. Xia and W. Caminati, Conformational Equilibria in Carboxylic Acid Bimolecules: A Rotational Study of Acrylic Acid-Formic Acid, Phys. Chem. Chem. Phys., 2013, 15, 2917-2922.

9 Q. Gou, G. Feng, L. Evangelisti and W. Caminati, Conformational Equilibria in Bimolecules of Carboxylic Acids: A Rotational Study of Fluoroacetic Acid-Acrylic Acid, J. Phys. Chem. Lett., 2013, 4, 2838-2842.

10 Q. Gou, G. Feng, L. Evangelisti and W. Caminati, A Rotational Study of cis and trans Acrylic Acid-Trifluoroacetic Acid, J. Phys. Chem. A, 2013, 117, 13500-13503.

11 Q. Gou, G. Feng, L. Evangelisti and W. Caminati, Conformers of Dimers of Carboxylic Acids in the Gas Phase: A Rotational Study of Difluoroacetic Acid-Formic Acid, Chem. Phys. Lett., 2014, 591, 301-305.

12 G. Feng, Q. Gou, L. Evangelisti and W. Caminati, Frontiers in Rotational Spectroscopy: Shapes and Tunneling Dynamics of the Four Conformers of the Acrylic Acid-Difluoroacetic Acid Adduct, Angew. Chem., Int. Ed., 2014, 53, 530-534.

13 L. Evangelisti, G. Feng, Q. Gou and W. Caminati, The Rotational Spectrum of Formic Acid...Fluoroacetic Acid, J. Mol. Spectrosc., 2014, 299, 1-5.

14 Q. Gou, G. Feng, L. Evangelisti and W. Caminati, Conformational Equilibria and Large Amplitude Motions in Dimers of Carboxylic Acids: The Rotational Spectrum of Acetic Acid-Difluoroacetic acid, ChemPhysChem, 2014, 15, 2977-2984.

15 D. Priem, T.-K. Ha and A. Bauder, Rotational Spectra and Structures of Three Hydrogen-Bonded Complexes between Formic Acid and Water, J. Chem. Phys., 2000, 113, 169-175.

16 B. Ouyang, T. G. Starkey and B. J. Howard, High-Resolution Microwave Studies of Ring-Structured Complexes between Trifluoroacetic Acid and Water, J. Phys. Chem. A, 2007, 111, 6165-6175.

17 B. Ouyang and B. J. Howard, High-Resolution Microwave Spectroscopic and ab initio Studies of Propanoic Acid and Its Hydrates, J. Phys. Chem. A, 2008, 112, 8208-8214.

18 B. Ouyang and B. J. Howard, The Monohydrate and Dihydrate of Acetic Acid: A High-Resolution Microwave Spectroscopic Study, Phys. Chem. Chem. Phys., 2009, 11, 366-373.

19 B. Ouyang and B. J. Howard, Hydrates of trans- and gaucheDifluoroacetic Acids: A High-Resolution Microwave Spectroscopic Study, J. Phys. Chem. A, 2010, 114, 4109-4117.
20 E. G. Schnitzler and W. Jäger, The Benzoic Acid-Water Complex: A Potential Atmospheric Nucleation Precursor Studied Using Microwave Spectroscopy and ab initio Calculations, Phys. Chem. Chem. Phys., 2014, 16, 2305-2314.

21 G. Feng, Q. Gou, L. Evangelisti, L. Spada, S. Blanco and W. Caminati, Hydrated Forms of Fluoroacetic Acid: A Rotational Study", Phys. Chem. Chem. Phys., 2016, 18, 23651-23656.

22 A. Vigorito, Q. Gou, C. Calabrese, S. Melandri, A. Maris and W. Caminati, How $\mathrm{CO}_{2}$ Interacts with Carboxylic Acids: A Rotational Study of Formic Acid- $\mathrm{CO}_{2}$, ChemPhysChem, 2015, 16, 2961-2967.

23 Q. Gou, L. B. Favero, S. S. Bahamyirou, Z. N. Xia and W. Caminati, Interactions between Carboxylic Acids and Aldehydes: A Rotational Study of $\mathrm{HCOOH}-\mathrm{CH}_{2} \mathrm{O}, J$. Phys. Chem. A, 2014, 118, 10738-10741.

24 A. M. Daly, B. A. Sargus and S. G. Kukolich, Microwave Spectrum and Structural Parameters for the FormamideFormic Acid Dimer, J. Chem. Phys., 2010, 133, 174304.

25 L. Evangelisti, L. Spada, W. Li, A. Ciurlini, J.-U. Grabow and W. Caminati, Shape of the Adduct Formic Acid-Dimethyl Ether: A Rotational Study, J. Phys. Chem. A, 2016, 120, 2863-2867.

26 R. B. Mackenzie, C. T. Dewberry and K. R. Leopold, The Trimethylamine-Formic Acid Complex: Microwave Characterization of a Prototype for Potential Precursors to Atmospheric Aerosol, J. Phys. Chem. A, 2016, 120, 2268-2273.

27 L. Spada, Q. Gou, B. M. Giuliano and W. Caminati, Interactions between Carboxylic Acids and Heteroaromatics: A Rotational Study of Formic Acid-Pyridine, J. Phys. Chem. A, 2016, 120, 5094-5098.

28 W. Caminati, A. Millemaggi, J. L. Alonso, A. Lesarri, J. C. López and S. Mata, Molecular Beam Fourier Transform Microwave Spectrum of the Dimethylether-Xenon Complex: Tunnelling Splitting and ${ }^{131}$ Xe Quadrupole Coupling Constants, Chem. Phys. Lett., 2004, 392, 1-6.

29 T. J. Balle and W. H. Flygare, Fabry-Perot Cavity Pulsed Fourier Transform Microwave Spectrometer with a Pulsed Nozzle Particle Source, Rev. Sci. Instrum., 1981, 52, 33-45.

30 J.-U. Grabow, W. Stahl and H. Dreizler, A Multioctave Coaxially Oriented Beam-Resonator Arrangement FourierTransform Microwave Spectrometer, Rev. Sci. Instrum., 1996, 67, 4072-4084.

31 J. L. Neill, S. T. Shipman, L. Alvarez-Valtierra, A. Lesarri, Z. Kisiel and B. H. Pate, Rotational Spectroscopy of Iodobenzene and Iodobenzene-Neon with a Direct-Digital 2-8 GHz Chirped-Pulse Fourier Transform Microwave Spectrometer, J. Mol. Spectrosc., 2011, 269, 21-29.

32 M. J. Frisch, G. W. Trucks, H. B. Schlegel, G. E. Scuseria, M. A. Robb, J. R. Cheeseman, G. Scalmani, V. Barone, B. Mennucci, G. A. Petersson, H. Nakatsuji, M. Caricato, X. Li, H. P. Hratchian, A. F. Izmaylov, J. Bloino, G. Zheng, J. L. Sonnenberg, M. Hada, M. Ehara, K. Toyota, R. Fukuda, J. Hasegawa, M. Ishida, T. Nakajima, Y. Honda, O. Kitao, H. Nakai, T. Vreven, J. A. Montgomery Jr, J. E. Peralta, F. Ogliaro, M. Bearpark, J. J. Heyd, E. Brothers, K. N. Kudin, V. N. Staroverov, T. Keith, R. Kobayashi, J. Normand, K. Raghavachari, A. Rendell, J. C. Burant, S. S. Iyengar, 
J. Tomasi, M. Cossi, N. Rega, J. M. Millam, M. Klene, J. E. Knox, J. B. Cross, V. Bakken, C. Adamo, J. Jaramillo, R. Gomperts, R. E. Stratmann, O. Yazyev, A. J. Austin, R. Cammi, C. Pomelli, J. W. Ochterski, R. L. Martin, K. Morokuma, V. G. Zakrzewski, G. A. Voth, P. Salvador, J. J. Dannenberg, S. Dapprich, A. D. Daniels, O. Farkas, J. B. Foresman, J. V. Ortiz, J. Cioslowski and D. J. Fox, Gaussian 09, Revision D.01, Gaussian, Inc., Wallingford CT, 2013.

33 J. L. Alonso, R. Spielh, A. Guarnieri, J. C. López and A. Lesarri, The Centimeter and Millimeter Microwave Spectrum of Cyclobutanone, J. Mol. Spectrosc., 1992, 156, 341-359.

34 S. Melandri, A. Maris, B. M. Giuliano and W. Caminati, Water-Ketones Hydrogen Bonding: The Rotational Spectrum of Cyclobutanone-Water, J. Chem. Phys., 2005, 123, 164304.

35 P. Ottaviani, W. Caminati, L. B. Favero, S. Blanco, J. C. López and J. L. Alonso, Molecular Beam Rotational Spectrum of Cyclobutanone-Trifluoromethane: Nature of Weak $\mathrm{CH} \cdots \mathrm{O}=\mathrm{C}$ and CH $\cdots$ F Hydrogen Bonds, Chem. - Eur. J., 2006, 12, 915-920.

36 H. M. Pickett, Vibration-Rotation Interactions and the Choice of Rotating Axes for Polyatomic Molecules, J. Chem. Phys., 1972, 56, 1715-1723.

37 H. M. Pickett, The Fitting and Prediction of VibrationRotation Spectra with Spin Interactions, J. Mol. Spectrosc.,
1991, 148, 371-377. Current versions are described and available at URL: http://spec.jpl.nasa.gov.

38 J. K. G. Watson, in Vibrational Spectra and Structure, ed. J. R. Durig, Elsevier, New York/Amsterdam, 1977, vol. 6, pp. 1-89.

39 J. Kraitchman, Determination of Molecular Structure from Microwave Spectroscopic Data, Am. J. Phys., 1953, 21, 17-25. 40 A. R. Ubbelohde and K. J. Gallagher, Acid-Base Effects in Hydrogen Bonds in Crystals, Acta Crystallogr., 1955, 8, 71-83.

41 R. Meyer, Flexible Models for Intramolecular Motion, a Versatile Treatment and Its Application to Glyoxal, J. Mol. Spectrosc., 1979, 76, 266-300.

42 H. M. Pickett and H. L. Strauss, Conformational structure, energy, and inversion rates of cyclohexane and some related oxanes, J. Am. Chem. Soc., 1970, 92, 7281-7290.

43 D. J. Millen, Determination of Stretching Force Constants of Weakly Bound Dimers from Centrifugal Distortion Constants, Can. J. Chem., 1985, 63, 1477-1479.

44 S. E. Novick, S. J. Harris, K. C. Janda and W. Klemperer, Structure and Bonding of KrClF: Intermolecular Force Fields in van der Waals Molecules, Can. J. Phys., 1975, 53, 2007-2015. 